\title{
Review of Graphene Supercapacitors and Different Modified Graphene Electrodes
}

\author{
Chenyuhu Yang \\ Chemistry Department, Xiamen University, Xiamen, China \\ Email: 20420172201961@stu.xmu.edu.cn
}

How to cite this paper: Yang, C. (2021) Review of Graphene Supercapacitors and Different Modified Graphene Electrodes. Smart Grid and Renewable Energy, 12, 1-15. https://doi.org/10.4236/sgre.2021.121001

Received: November 10, 2020

Accepted: January 26, 2021

Published: January 29, 2021

Copyright ( 2021 by author(s) and Scientific Research Publishing Inc. This work is licensed under the Creative Commons Attribution International License (CC BY 4.0).

http://creativecommons.org/licenses/by/4.0/

\begin{abstract}
Faced with the exhaustion of traditional energy sources, the development of new energy devices is the current priority. Supercapacitor is a new type of energy storage device with high power density and long cycle life. Carbon material, metal oxide and conductive polymer are three common electrode materials of supercapacitors. Based on China's energy situation, this paper puts forward new ideas from coal to graphene to supercapacitors. In addition, the research progress of graphene supercapacitors is summarized, including the principle of supercapacitors, preparation methods from coal to graphene, graphene electrode materials and different modification, and some possible scientific problems in the research process of different modified graphene electrodes are also reviewed and prospected.
\end{abstract}

\section{Keywords}

Supercapacitor, Energy Sources

\section{Introduction}

Faced with the pollution and gradual exhaustion of traditional energy, the development of new energy is an effective supplement to the whole energy supply system and an important measure to control and protect the environment [1]. The development of new energy has become an important task to be solved in society today. Recently, while people are actively looking for clean and efficient renewable energy, they are also focusing on energy storage, especially electric energy storage. Supercapacitor research has been a heated topic. Supercapacitor is a new type of energy storage device between traditional capacitor and rechargeable battery. Supercapacitors have long cycle life, simple mechanism, strong dynamic charge propagation, high specific energy and high specific power [2]. 
Compared with traditional capacitors, supercapacitors have higher power capacity and higher energy density. At present, supercapacitors are widely used in consumer electronics, storage systems and industrial power and energy management. According to the mechanism by which electricity is stored, supercapacitors can be divided into electric double layer capacitors (EDLC) and Pesudocapacitors.

The electric double layer capacitor is a new type of component that stores energy through the interface double layer formed between the electrode and electrolyte. When the electrode and electrolyte contact, due to the effect of coulomb force, intermolecular force and interatomic force, a stable double layer charge with opposite sign appears at the solid-liquid interface, which is called the interface double layer [3]. The electrode materials used by the double layer capacitor are mostly porous carbon materials, including activated carbon (activated carbon powder, activated carbon fiber), carbon aerogel, and carbon nanotubes. The capacity of double layer capacitor is related to the porosity of electrode material. Generally, the higher the porosity is, the greater specific surface area of the electrode material is and the greater the double layer capacity is.

Pseudcapacitors, also known as Faraday quasi-capacitors, are capacitors related to electrode charging potential when electroactive substances undergo low potential deposition on the surface of electrode materials or two-dimensional or quasi-two-dimensional space of body phase through highly reversible absorption/desorption or oxidation/reduction [4]. The capacity of a pseudocapacitor is 10 to 100 times that of an EDLC of the same volume or weight. At present, the material of pseudo capacitor electrode mainly consists of some metal oxides and conductive polymers.

At present, many carbon-based materials are commonly used as electrodes for supercapacitors. Among them, graphene, as a new two-dimensional carbon nanostructure, has a large specific surface area and an unusually high electron mass [5]. If large scale production of graphene is possible, it will have attractive potential in the field of supercapacitors [6]. The structure of the polyaromatic hydrocarbons in coal has a narrow particle size distribution, and the structure of asphalt is very similar to that of graphene, including the $\mathrm{sp}^{2}$ hybridization. This property makes it possible to synthesize graphene directly from coal tar asphalt.

In addition, China is one of the few countries in the world that uses coal as its main energy source [7] and already produces more than a third of coal in the world, importing most of its oil by contrast [8]. Therefore, according to China's energy condition and structure, rich in coal and poor in oil, it is of great significance and value to develop graphene supercapacitor electrodes made from coal. This paper summarizes some methods of preparing graphene from coal and some development in graphene supercapacitors at first and then compares several different compounds modifying or doping on the graphene electrodes of supercapacitors, and most importantly proposes a brand new idea, from coal to graphene to supercapacitor throughout the whole article. 


\section{Graphene from Coal}

First of all, this article introduces how coal is formed and the composition of coal. Coal is an heterogeneous rock dominated by organic matter physically and chemically. It is generally believed that coal is an extremely thick layer of black humus accumulated on the ground by the branches, leaves and roots of plants over hundreds of thousands of years. In addition, coals are constantly buried underground due to the changes in the crust of the earth and isolated from the air for a long time, forming by a series of complex physical and chemical changes under high temperature and pressure [9].

The development and utilization of coal have been going on for hundreds of years, and the discovery that coal has the potential to make graphene is very exciting for scientists. Several techniques have been developed for making graphene from coal, for example, hydrazine chemical redox, bitumen for heat treating coal tar (when aluminum is used as a catalyst), and oxidation extraction (coal is oxidized with nitric acid and then extracted with $\mathrm{NaOH}$ ). In 2012, Zhou group synthesized chemically derived graphene and noble metal composite materials by catalytic graphitization, chemical oxidation and dielectric barrier discharge (DBD) plasma-assisted deoxidation using coal as the starting material. They studied the electrochemical properties of the material as an electrode for supercapacitors, demonstrating its potential for energy storage and environmental protection [10]. In 2014, Xu research group of Fuzhou University proposed an effective method for the preparation of graphene nanoscale sheets by high temperature pyrolysis of asphalt with the catalysis of aluminum [11]. And then, Gao research group proposed that using coal derived graphite (CDG) as carbon source, $\mathrm{MnSO}_{4}$ was converted into $\mathrm{Mn}_{3} \mathrm{O}_{4}$ in situ through precipitation in the air, and then reduced by hydrazine. Observably, the reduced coal derived graphene $/ \mathrm{Mn}_{3} \mathrm{O}_{4}$ was obtained and used as electrode material for supercapacitors, with high atomic efficiency, considerable specific capacitance and energy density [12].

It is worth mentioning that in the same year, He research group synthesized 3D hollow porous graphene balls (HPGBs) by using coal tar pitch as raw material, adopting simple nanometer $\mathrm{MgO}$ template strategy and using $\mathrm{KOH}$ as activator. The simple production process is shown in Figure 1. As a supercapacitor electrode, HPGBs shows excellent specific capacitance, and cycle life [13]. To explore other possible synthetic methods, in 2016, Das research group presented the oxidation extraction method. In their research, coal is first oxidized by nitric acid, and then extracted in an alkaline solution of sodium hydroxide. During oxidative extraction, they observed the formation of coal derived carbon nanomaterials and some clusters of graphene nanoflakes [14]. In 2017, He team proposed a completely new synthesis method using nano $\mathrm{ZnO}$ template strategy and combined with $\mathrm{KOH}$ in situ activation technology to prepare 3D interconnected graphene nanocapsules from a variety of aromatic hydrocarbons. This $3 \mathrm{D}$ porous network provides a pathway for good electron conduction and a rich microhole for ion adsorption. As the electrode of supercapacitor, it also has high 


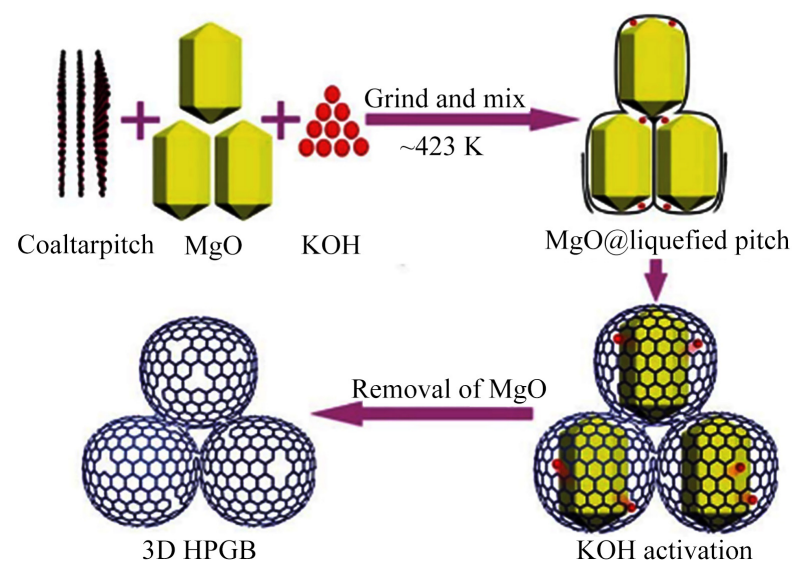

Figure 1. Schematic diagram of direct preparation of 3D HPGB from coal tar pitch [13].

capacitance and excellent cycle life [15]. All this work mentioned above could possibly pave the way for more efficient mass production of low-cost graphene made from coal.

\section{Graphene Supercapacitors}

Mitigating or solving energy problems requires good efficiency at every step in the production line. Given the graphene made from coal, the next step is developing techniques and methods to use and text its electrochemical performance as electrode for supercapacitor. Many researchers have made contributions to this field. Considering the characteristics of graphene and requirements of consumers for electronic devices, scientists hope to develop and design graphene supercapacitors to be more flexible. Maher's team at the University of California, Los Angeles, United States, used a direct laser method to get graphene in 2012. The film is mechanically robust with high electrical conductivity and specific surface area, so it can be used directly as electrodes. The devices made with these electrodes exhibit ultrahigh energy density in different electrolytes, maintaining high power density and good cycling stability as well. In addition, these materials are with excellent electrochemical properties under high mechanical stress, so they are expected to become high power flexible electronic devices [16]. In 2013, they went a step further and developed a tiny graphene supercapacitor that not only looks small and flexible, but can charge a cell phone or even a car 1,000 times faster than a normal battery (Figure 2).

Another development worth mentioning is Liu's work. Using plain cotton cloth as a platform and a stable GO suspension as ink, Liu's team prepared a flexible and easily processed "brush drying" process. After this simple manufacturing operation, then annealed in argon at $300^{\circ} \mathrm{C}$, the resulting graphene-cotton composite fabric has good electrical conductivity, excellent flexibility, and strong adhesion. As the electrode of supercapacitor, the specific capacitance in aqueous solution is satisfactory [17]. In the same year, the Dong team used a simple hydrothermal method to synthesize cobalt oxide in situ on a 3D graphene foam 


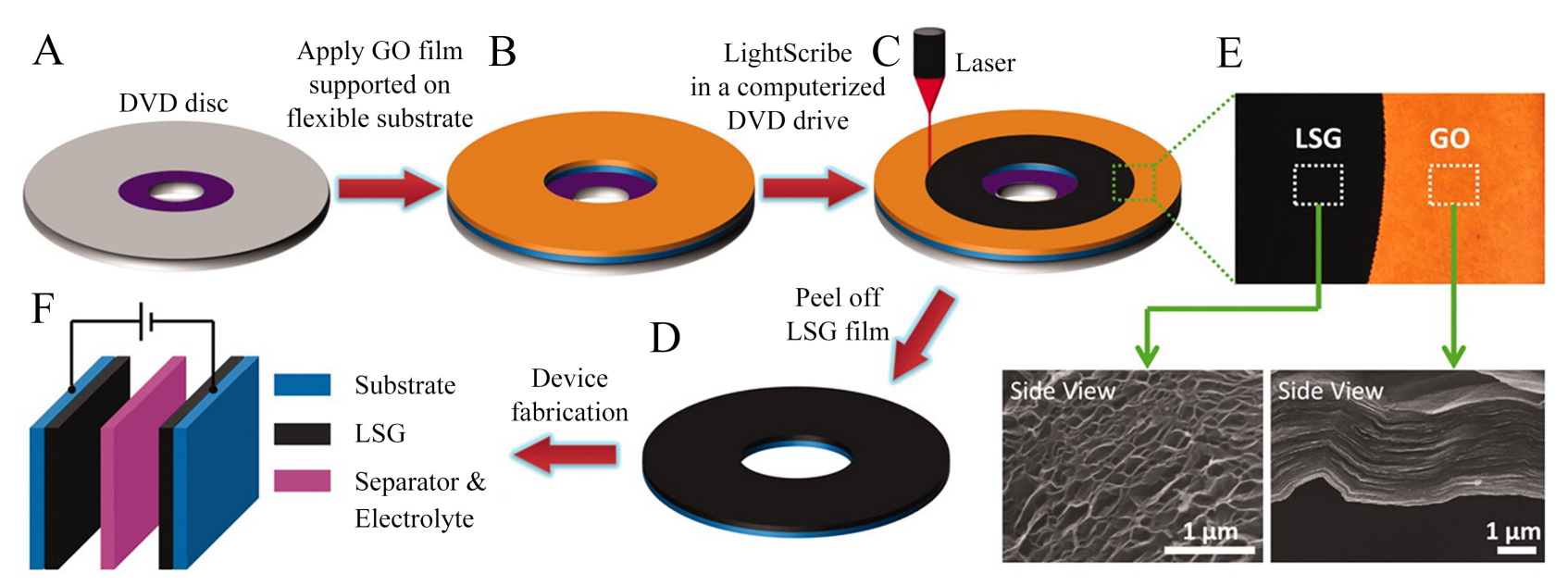

Figure 2. Schematic diagram of fabrication of electrochemical capacitor of graphene by laser engraving [16].

grown by chemical vapor deposition. The 3D graphene/cobalt oxide composite was used as a single-chip independent electrode for supercapacitor applications and glucose enzyme electrolysis detection. Distinctly, they demonstrated that it provided high specific capacitance and very good cyclic stability [18].

All the research above is exciting developments in the field. Having attaining so many excellent achievements, scientists also pay attention to some difficulties and unsolved problems for graphene electrodes of supercapacitors. Due to the fact that graphene is easy to aggregate, it is difficult for graphene to reach the theoretical specific surface area and conductivity. Therefore, the research on graphene is focusing on ensuring and enlarging the specific surface area of graphene and obtaining an appropriate pore diameter distribution. The most considerable modifying methods are to combine with other materials or dope impurity atoms. Next, some different methods of modifying graphene supercapacitors are summarized and compared.

\section{N-Doped Graphene Electrodes}

In general, the atoms that can be used to dope graphene include nitrogen, boron, phosphorus, and so on. These doping atoms can open band gap and adjust the conductive type, change the electronic structure of graphene, improve the density of free carriers, and thus improve the conductivity and stability of graphene. Among them the nitrogen doped graphene is the most studied one. When doped with nitrogen atoms, three bonding types are usually formed in the lattice of carbon atoms: Pyridinic-N, Pyrrolic-N, and graphitic-N. Pyridine-N refers to the nitrogen atoms attached to two carbons on the edge of the graphite surface. In addition to providing an electron to the conjugated bond system, the nitrogen atoms also have a pair of lone electrons, which can absorb oxygen molecules and their intermediates during the oxygen reduction process. Pyrrolic- $\mathrm{N}$ is a nitrogen atom conjugated with two $\mathrm{p}$ electrons and ambond system. Graphitic-N represents nitrogen attached to three carbon atoms on the graphite base surface [19] (Figure 3). 


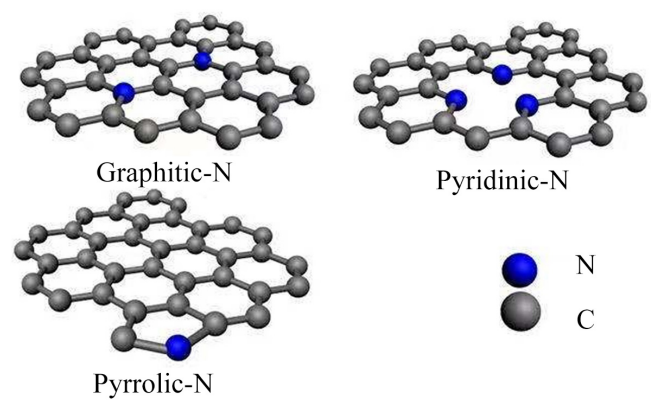

Figure 3. Schematic diagram of nitrogen doped graphene.

There are many nitrogen doping methods, including hydrothermal method, chemical vapor deposition, nitrogen plasma discharge method, arc discharge method and some of them are discussed below.

Chemical vapor deposition (CVD) is a method that uses the precursor reactants in the gaseous state to decompose some components of the precursor through the chemical reaction between atoms and molecules to form a thin film on the matrix. In 2010, Dai's research group introduced $\mathrm{NH}_{3}$ into methane chemical vapor deposition to synthesize nitrogen-doped graphene showing great electrochemical performance [20]. Wei team obtained nitrogen-doped graphene by chemical vapor deposition (CVD) with copper as catalyst and methane and ammonia as carbon source and nitrogen source respectively under an atmosphere of hydrogen gas at $800^{\circ} \mathrm{C}$. The highlight of this work is that in the process of CVD, the doped composite graphene with carbon atoms can be replaced by $\mathrm{N}$ atoms in the graphene lattice. This substitution hybrid can effectively regulate the electrochemical properties of graphene [21] (Figure 4).

After that, Su group explored another way of doping nitrogen and synthesized nitrogen doped graphene by hydrothermal method using graphene oxide (GO) as raw material and urea as reduction dopant. They found that along with the reduced go flakes, nitrogen was also doped into the graphene plane. They also tested the electrochemical properties of graphene. Nitrogen-doped graphene with a nitrogen content of $7.50 \%$ has excellent capacitance and long cycle life. Moreover, the first cycle specific discharge capacitance of the supercapacitor electrode is $184.5 \mathrm{~F} \cdot \mathrm{g}^{-1}$, which has great practical potential [22].

Nitrogen plasma discharge (NPD) and arc discharge (AD) are also effective methods for the synthesis of nitrogen-doped graphene. NPD uses $\mathrm{N}^{+}, \mathrm{N}_{2}$ and $\mathrm{NH}_{3}$ plasmas to radiate graphene and then gets nitrogen-doped graphene by annealing or thermal stripping. In 2010, Guo's team treated $\mathrm{N}^{+}$irradiated graphene using $\mathrm{NH}_{3}$. They reported the graphene obtained from nitrogen-doped graphene samples irradiated by $\mathrm{N}^{+-}$ions and annealed by ammonia [23]. AD is a method to generate NG by arc discharge between carbon electrodes in the atmosphere of hydrogen and nitrogen source. Using ammonia or pyridine as nitrogen sources, Panchakarla generates two or three layers of graphene by conducting an arc discharge between carbon electrodes in an atmosphere of hydrogen [24]. 


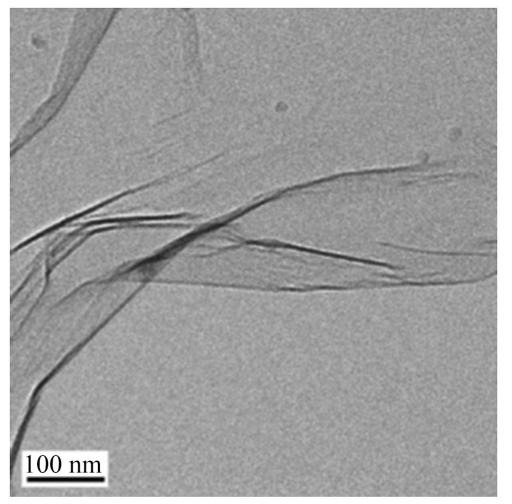

Figure 4. TEM image of nitrogen doped graphene.

To sum up, many scientists have made their contributions in this field, but they still can't break through some difficulties. An important drawback or difficulty is that there is a frustrating limit to the amount of nitrogen-doped graphene. And how to control the type and distribution of nitrogen doping without greatly reducing the electron mobility remains to be solved. What is more, it is still a challenge to achieve the specific location of nitrogen doping and precise control of nitrogen doping to ensure higher electrochemical performance. Of course, there is one point that cannot be ignored, the method of synthesizing NG on a large scale remains unsolved. As a result of all the facts mentioned above, the nitrogen doped graphene electrode of supercapacitor is still at its early stage now, but the scientific search future for it is worth looking forward to.

\section{Graphene/Metal Oxide Electrodes}

Among the graphene/metal oxide composites, graphene and metal oxide can give full play to their respective advantages. Combined with the excellent cycling stability performance of graphene and the high capacity characteristics of metal oxide, the comprehensive performance of nanocomposites can be greatly improved. The nanoparticles are embedded between adjacent graphene sheets, effectively preventing the sheets from restacking and allowing the high charge capacity to be retained to compensate for the lack of graphene as an electrode material for pseudcapacitors [25]. Then, some of the most studied graphene/metal oxide electrodes in recent years will be shown and discussed.

The low conductivity and load rate of $\mathrm{MnO}_{2}$ limit its application in supercapacitors. But, the combination of $\mathrm{MnO}_{2}$ and graphene can compensate the shortcomings of both $\mathrm{MnO}_{2}$ and graphene which is easy to aggregate. Scientists have explored many ways of preparing graphene/ $\mathrm{MnO}_{2}$ composites, mainly including solvothermal (hydrothermal) method, electrochemical deposition method, chemical reaction method and self-assembly method. Depositing $\mathrm{MnO}_{2}$ particles onto a porous "activated microwave expanded graphite oxide" (aMEGO), Zhao research group prepared granular $\mathrm{MnO}_{2}$ /aMEGO (AGMn) by means of self-controlled redox process using $\mathrm{KOH}$ as activator. AMEGOs are used as the electrodes of the supercapacitors. Tests show that the maximum energy density 
and maximum power density of the system can reach $24.3 \mathrm{Wh} \cdot \mathrm{kg}^{-1}$, and the capacitance can be maintained at $80 \%$ after 5000 cycles [26] (Figure 5).

Li's team prepared the granular $\mathrm{MnO}_{2} /$ graphene composite using the prepared graphene, $\mathrm{KMnO}_{4}$ and $\mathrm{K}_{2} \mathrm{SO}_{4}$ as raw materials. Using $1 \mathrm{M} \mathrm{Na}_{2} \mathrm{SO}_{4}$ aqueous solution as electrolyte, the electrochemical properties of the composite electrode were studied by cyclic voltammetry and electrochemical impedance spectroscopy. When the potential scanning rate is $2 \mathrm{mV} \cdot \mathrm{s}^{-1}$, its specific capacitance value reaches $211.5 \mathrm{~F} \cdot \mathrm{g}^{-1}$, and the electrode shows good cycling performance and capacitance value [27] (Figure 6).

Another metal oxide of great research value is $\mathrm{RuO}_{2}$. It is one of the earliest transition metal oxides to be found and studied as $\mathrm{RuO}_{2}$ itself has good electrical conductivity, high capacity and small internal resistance, which shows excellent potential traits as supercapacitor electrode and could compensate for the drawbacks of graphene. However, due to its scarcity and high costs, scores of researches are going into the efforts to reduce the cost of the preparation and the amount of novel metals. One of the significant ways is to combine with the graphene as composite. The preparation method of $\mathrm{RuO}_{2} /$ graphene composites that have been greatly developed is sol gel method. Generally, sol-gel method dissolves graphene and $\mathrm{RuCl}_{3} \cdot \mathrm{xH}_{2} \mathrm{O}$ in solution, then adds $\mathrm{NaOH}$ and other alkaline aqueous solutions. After agitation and filtration, the precursor is obtained, and then the product is prepared by heat treatment. The Rakhi team successfully used metal oxide nanoparticles as supercapacitor electrodes by chemically attaching them to graphene nanosheets (GNs) and using the synthetic material coated on a conductive carbon substrate [28]. This method improves the electrochemical performance due to the further optimization of graphene, and the results show that the addition of metal oxide nanoparticles improves the capacitive performance of GNs. The detection of the supercapacitor found that when the scanning rate was $20 \mathrm{mV} \cdot \mathrm{s}^{-1}$, the maximum initial specific capacitance value was increased to $365 \mathrm{~F} \cdot \mathrm{g}^{-1}$, and the energy density was $50.6 \mathrm{Wh} \cdot \mathrm{kg}^{-1}$. These findings indicate the importance and great potential of metal oxide /GNs composite coated carbon fabrics in high performance development.

$\mathrm{NiO}$ as the electrode of supercapacitor has been studied a lot as well, for the reason that it has the advantages of high theoretical pseudocapacitance and good cyclic reversibility. By combining $\mathrm{NiO}$ with graphene, not only the easy aggregation of graphene can be avoided, but a supercapacitor electrode with excellent electrochemical performance also can be obtained. In 2014, Wu using nickel foam as template got graphene with three dimensional network structure, and then put it in the $\mathrm{NiSO}_{4}$ and ammonia solution to make the $\mathrm{Ni}(\mathrm{OH})_{2}$ on the graphene bubble grow. Finally after high temperature heat decomposition, $\mathrm{Ni}$ $(\mathrm{OH})_{2}$ was flaked as $\mathrm{NiO} /$ graphene composites. And then, the electrochemical properties in the three-electrode system were tested [29]. The simple synthetic method is shown in Figure 7. Because of the high degree of graphitization, the structural scaffold has high conductivity, which effectively promotes the electron 


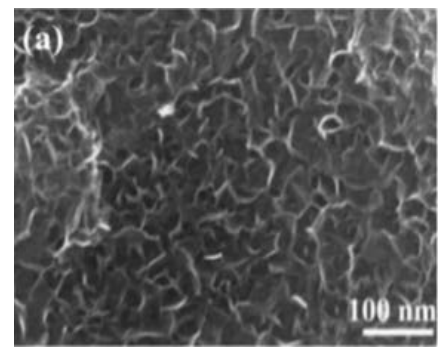

Figure 5. High resolution SEM image of AGMn-120 min [26].

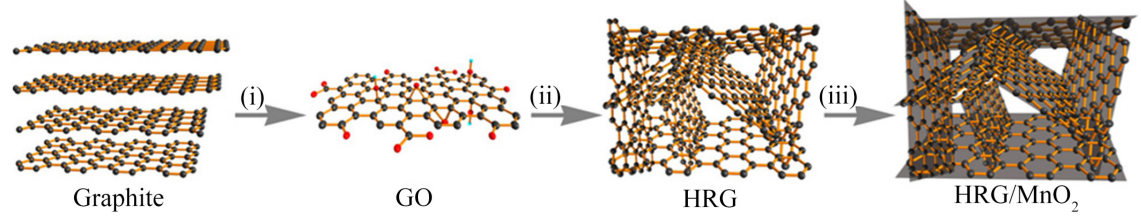

Figure 6. Schematic diagram of $\mathrm{HRG} / \mathrm{MnO}_{2}$ synthesis [27].

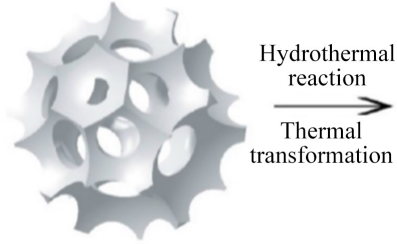

Ni foam

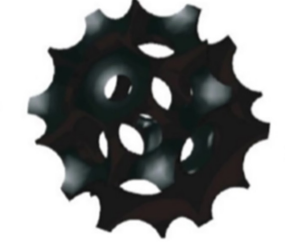

3D UDG/NF scaffold

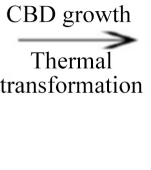

3D NiO/UDG/HF hybrid 난 $\mathrm{NiO}(\mathrm{CBD})$

Figure 7. Schematic diagram of $\mathrm{NiO} / \mathrm{G} / \mathrm{NF}$ synthesis [29].

transmission to $\mathrm{NiO}$. It is found that the complex is a $3 \mathrm{D}$ network structure, and the specific capacitance value is $42 \mathrm{~F} \cdot \mathrm{g}^{-1}$ when the current density is $2 \mathrm{~A} \cdot \mathrm{g}^{-1}$. Moreover, the cycle stability is excellent, after 500 charge and discharge cycles, the capacitance retention rate is $90 \%$.

In summary, the types and morphologies of metal oxides in the composites, the preparation methods, the structure and dispersion of graphene, all have prominent influence on their electrochemical performance. Based on the existing research in this field, scientists suggest several directions that deserve further research. First, the morphology of the metal compounds in the composites needs to be controlled and optimized to improve the electrochemical performance. Secondly, the structure of graphene should be modified more precisely according to needs, such as using template method to make graphene sheets with large pore structure, or using CVD method to prepare graphene with three-dimensional structure, changing the distance between graphene sheets, using surfactant to modify graphene to improve its dispersibility and adsorbability.

\section{Graphene/Conductive Polymer Electrodes}

In addition to doping other atoms and compounding with metal oxides, the combination of graphene and conductive polymers is also a very effective and promis- 
ing way to enhance the performance of graphene as electrode of supercapacitor. The composites of graphene and conductive polymer, which both have large delocalized conjugate system, can realize the complementary properties of these two functional materials to some extent, and could further enhance and expand the properties of the materials by utilizing the electron transfer between them. On the other hand, the combination can also overcome the inconvenience process caused by the small size of graphene nano sheets and avoid to lose the excellent properties of single layer graphene caused by stacking between graphene nano sheets at the same time.

On the whole, the preparation of graphene/conductive polymer composites includes solution blending, in situ polymerization and electrochemical polymerization. Solution blending is the simplest method to prepare graphene and conductive polymer composites. It requires the graphene materials and conductive polymers to be steadily dispersed in common solvents. Shi's team in Tsinghua University prepared a composite film (G-PNF) by vacuum filtration using a mixture of chemically converted graphene (CCG) and polyaniline nanofibers (PANI-NFS) [30]. The composite film shows a structured "sandwich" layered structure with PANI-NFS sandwiched between CCG layers. In addition, it has mechanical stability and high flexibility. The electrostatic interaction between polyaniline fibers and graphene sheets not only reduces the interface repulsion effect, but also enhances the interaction between the two. As a result, the resultant composite films show certain mechanical strength and flexibility, so that they can be bent into large angles or molded into various required structures. It also has excellent supercapacitor properties $\left(210 \mathrm{~F} \cdot \mathrm{g}^{-1}\right)$ (Figure 8).

Another method of synthesizing graphene/conductivepolymers is In situ polymerization, in which the conductive polymer monomer is first uniformly dispersed into the system containing the graphene material and then the monomer polymerization is initiated. Due to electrostatic attraction, $\pi-\pi$ stacking, hydrogen or covalently bonded interactions, conductive polymer monomers are adsorbed or covalently modified on the graphene sheet surface, which is then polymerized by oxidants to grow on the graphene surface, thereby avoiding polymerization of monomers in solution. The advantage of this method is that it is possible to avoid the self-aggregation that occurs in the system when graphene is directly involved in in-situ polymerization. Wu's team in National University of Singapore first reported that chemically modified graphene and polyaniline nanofiber composites were prepared under acidic conditions using go in situ polymerization of aniline monomers [31]. The graphene oxide/polyaniline (PANI) composites with different mass ratios were reduced to graphene by hydrazine, and then the reduced PANI was reoxidized and reduced to obtain the graphene/PANI nanocomposites. The synthesis method is shown in Figure 9. The resulting material has a specific capacitance value of $480 \mathrm{~F} \cdot \mathrm{g}^{-1}$ at $0.1 \mathrm{~A} \cdot \mathrm{g}^{-1}$ charging current. This uniform structure, coupled with the observed high conductivity, gives it a high specific capacitance and good cycling stability. 


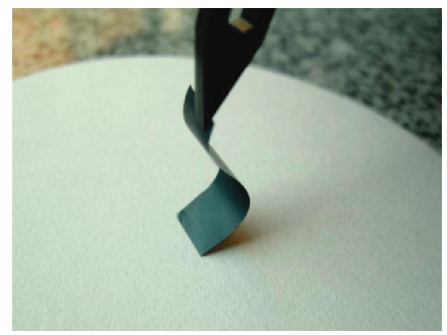

Figure 8. Photograph of a flexible G-PNF film [30].

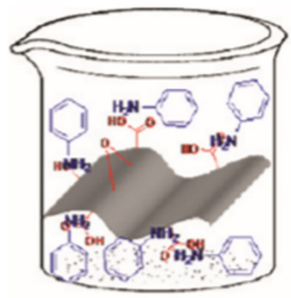

GO+Aniline

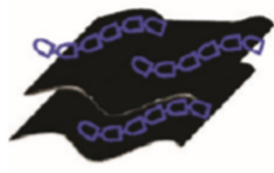

PAG

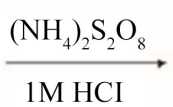

$\frac{\left(\mathrm{NH}_{4}\right)_{2} \mathrm{~S}_{2} \mathrm{O}_{8}}{1 \mathrm{M} \mathrm{HCI}}$

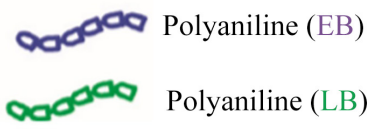

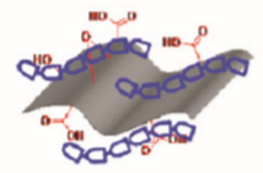

PAGO $95^{\circ} \mathrm{C} \downarrow \mathrm{NH}_{2} \mathrm{NH}_{2} \mathrm{H}_{2} \mathrm{O}$

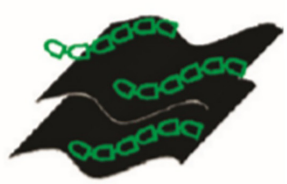

$$
\text { @od Polyaniline (LB) }
$$

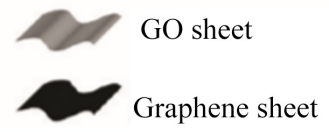

Figure 9. Schematic diagram of graphene-PANI synthesis [31].

Electrochemical polymerization is also an important method in this field. And studies have reported that carbon atoms have a network conduction structure in the carbon/polypyrrole nanocomposites, and the conductivity can be increased by tunneling effect, which makes the conductivity and thermal stability better than that of pure polypyrrole [32]. For this reason, the preparation of the composite materials based on graphene to obtain electrode materials with better performance has become a hot research topic in the field of supercapacitors. In 2017, Du research group used graphene prepared by wet chemical reduction as the base and used in situ electrochemical deposition method to make graphene/polypyrrole conductive composite supercapacitor electrode [33]. The results showed that the specific capacitance of the graphene/polypyrrole conductive composite electrode prepared in $0.2 \mathrm{~mol} \cdot \mathrm{L}^{-1}$ pyrrole solution was up to 388 $\mathrm{F} \cdot \mathrm{g}^{-1}$, showing excellent electrochemical performance of supercapacitors.

The electrochemical polymerization method can not only prepare composite films with high mechanical strength, but also precisely control the electrochemical polymerization process by adjusting electrochemical parameters, such as potential range and current density. In Wang group, graphene is obtained by the 


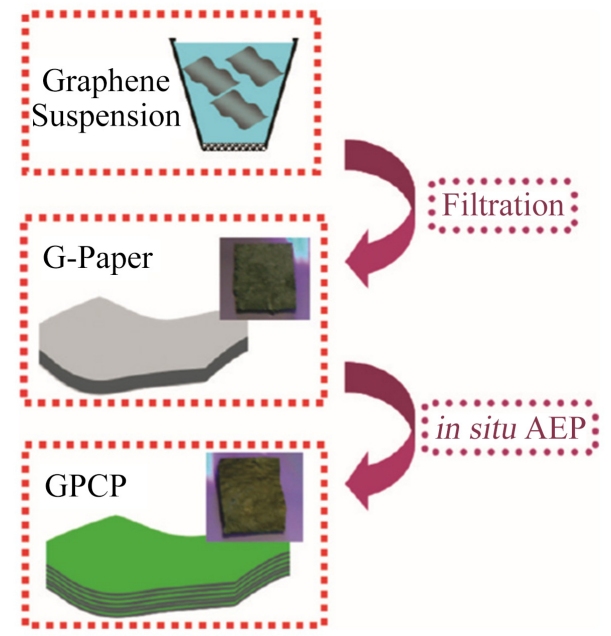

Figure 10. Schematic diagram of GPCP synthesis [34].

vacuum suction filter film as electrode, the containing water system of electrochemical polymerization of aniline monomer was prepared for the first time to the flexible graphene/polystyrene supported amine compound film. The graphene/polyaniline composite paper (GPCP) produced by situ anodic electropolymerization (AEP) in this paper shows good tensile strength of $12.6 \mathrm{MPa}$ and a stable large capacitance (233 F.g $\mathrm{g}^{-1}$ ), which is better than those of many other existing carbon based flexible electrode [34] (Figure 10).

To summarize, although scientists have developed many methods for preparing graphene/conductive polymer composite electrodes, they have found that there are some difficulties in the process. Due to the formation of graphene with polyethylene glycol of conductive polymer, the effective specific surface area of composites is small, which limits its application in electrode materials. Therefore, how to prepare a large number of graphene/conductive polymer composites with high conductivity, high specific surface area and controllable micro-nano structure is an urgent problem to be solved.

\section{Conclusions}

Graphene has a large specific surface area, excellent conductivity and thermal conductivity, and it is used as the electrode of the supercapacitor. The graphene supercapacitor prepared has the characteristics of high power, rapid charge and discharge, and strong cycle stability. At present, the research direction of graphene supercapacitors is mainly the optimization of electrode materials and the improvement of the performance of the whole capacitor. In order to further improve the electrochemical performance of supercapacitors, the specific surface area of graphene sheets was increased by chemical modification to facilitate the entry of electrolyte. The matrix of the supercapacitor is flexible matrix, and the electrolyte is polymer electrolyte or ionic electrolyte, so the flexible supercapacitor can be made and hopefully used in flexible and light electronic devices. Second, graphene can be combined with such materials as conductive polymers 
and metal oxides to produce supercapacitors that have the advantages of both. However, the development of this field is still in its infancy, and many related problems still need to be solved. Graphene materials themselves cannot be prepared in large quantities, which limits the industrial production of composite materials. In addition, due to the advantages of supercapacitors, they can be used in new energy, transportation, consumer electronics, industrial and other fields according to the size of capacity.

In order to meet the needs of energy and environment, new energy electric vehicles have gradually become the focus of applications. The trend of application is to combine supercapacitors and batteries to make up for the lack of energy density and power density. According to the actual progress of the industrialization of graphene, the current problem is how to obtain the low-cost mass production of large areas of graphene materials, so as to further exert the application of graphene materials in the field of supercapacitors.

Faced with the pollution and depletion of traditional energy, the development of new energy industry is an effective supplement to the whole energy supply system and an important measure to control and protect the environment. The research direction, from coal to graphene to supercapacitors, is a potentially effective approach. The solution for energy problem depends on national policy and national energy situation. China is a country with rich coal and poor oil, so it is imperative to develop coal chemistry research. In addition, China's coal price is relatively cheap, and China has many coal plants, so the problems of expensive raw materials and industrialization are relatively easy to solve. To sum up, combined with the development of China's energy situation in the world energy industry, this research direction may bring some new ideas and thoughts. From a global perspective, under the trend of depletion of non-renewable resources and low carbon environmental protection, the study of new energy materials is of great significance, and the study of high-performance supercapacitors has broad application prospects and strategic significance.

\section{Conflicts of Interest}

The author declares no conflicts of interest regarding the publication of this paper.

\section{References}

[1] Kötz, R. and Carlen, M. (2000) Principles and Applications of Electrochemical Capacitors. Electrochimica Acta, 45, 2483-2498.

https://doi.org/10.1016/S0013-4686(00)00354-6

[2] Pandolfe, A.G. and Hollenkamp, A.F. (2006) Carbon Properties and Their Role in Supercapacitors. Journal of Power Sources, 157, 11-27.

https://doi.org/10.1016/j.jpowsour.2006.02.065

[3] Raymundo-Piero, E., Kierzek, K., Machnikowski, J., et al. (2006) Relationship between the Nanoporous Texture of Activated Carbons and Their Capacitance Properties in Different Electrolytes. Carbon, 44, 2498-2507. 
https://doi.org/10.1016/j.carbon.2006.05.022

[4] Long, J.W., Swider, K.E., Merzbacher, C.I., et al. (1999) Voltammetric Characterization of Ruthenium Oxide-Based Aerogels and Other $\mathrm{RuO}_{2}$ Solids: The Nature of Capacitance in Nanostructured Materials. Langmuir, 15, 780-785. https://doi.org/10.1021/la980785a

[5] Wang, H.L., et al. (2010) $\mathrm{Ni}(\mathrm{OH})_{2}$ Nanoplates Grown on Graphene as Advanced Electrochemical Pseudocapacitor Materials. Journal of the American Chemical Society, 132, 7472-7477. https://doi.org/10.1021/ja102267j

[6] Miller, J.R., et al. (2010) Graphene Double-Layer Capacitor with ac Line-Filtering Performance. Science, 329, 1637-1639. https://doi.org/10.1126/science.1194372

[7] Xu, J.-L., et al. (2003) Green Technique in Coal Mining. Journal of China University of Mining \& Technology, 32, 343-348.

[8] Chunrong T, Department S I. (2006) Analysis of China's Oil Import \& Export in 2005. International Petroleum Economics.

[9] Haenel, M.W. (1992) Recent Progress in Coal Structure Research. Fuel, 71, 1211-1223. https://doi.org/10.1016/0016-2361(92)90046-Q

[10] Zhou, Q., Zhao, Z., Zhang, Y., et al. (2012) Graphene Sheets from Graphitized Anthracite Coal: Preparation, Decoration, and Application. Energy \& Fuels, 26, 5186-5192. https://doi.org/10.1021/ef300919d

[11] Xu, H., Lin, Q., Zhou, T., et al. (2014) Facile Preparation of Graphene Nanosheets by Pyrolysis of Coal-Tar Pitch with the Presence of Aluminum. Journal of Analytical and Applied Pyrolysis, 110, 481-485. https://doi.org/10.1016/j.jaap.2014.10.017

[12] Gao, F., Qu, J., Zhao, Z., et al. (2014) A Green Strategy for the Synthesis of Graphene Supported $\mathrm{Mn}_{3} \mathrm{O}_{4}$ Nanocomposites from Graphitized Coal and Their Supercapacitor Application. Carbon, 80, 640-650. https://doi.org/10.1016/j.carbon.2014.09.008

[13] He, X., Zhang, H., Zhang, H., et al. (2014) Direct Synthesis of 3D Hollow Porous Graphene Balls from Coal Tar Pitch for High Performance Supercapacitors. Journal of Materials Chemistry A, 2, 19633-19640. https://doi.org/10.1039/C4TA03323I

[14] Das, T., Boruah, P.K., Das, M.R., et al. (2016) Formation of Onion-Like Fullerene and Chemically Converted Graphene-Like Nanosheets from Low-Quality Coals: Application in Photocatalytic Degradation of 2-Nitrophenol. RSC Advances, 6, 35177-35190. https://doi.org/10.1039/C6RA04392E

[15] He, X., Li, X., Ma, H., et al. (2017) ZnO Template Strategy for the Synthesis of 3D Interconnected Graphene Nanocapsules from Coal Tar Pitch as Supercapacitor Electrode Materials. Journal of Power Sources, 340, 183-191. https://doi.org/10.1016/j.jpowsour.2016.11.073

[16] Maher, F., et al. (2012) Laser Scribing of High-Performance and Flexible Graphene-Based Electrochemical Capacitors. Science (New York, N. Y.), 335, 1326-1330. https://doi.org/10.1126/science.1216744

[17] Liu, W.W., Yan, X.B., Lang, J.W., et al. (2012) Flexible and Conductive Nanocomposite Electrode Based on Graphene Sheets and Cotton Cloth for Supercapacitor. Journal of Materials Chemistry, 22, 17245-17253. https://doi.org/10.1039/c2jm32659k

[18] Dong, X.C., Xu, H., Wang, X.W., et al. (2012) 3D Graphene-Cobalt Oxide Electrode for High-Performance Supercapacitor and Enzymeless Glucose Detection. ACS Nano, 6, 3206-3213. https://doi.org/10.1021/nn300097q

[19] Chen, X., He, D., et al. (2013) Nitrogen-Doped Graphene. Progress in Chemistry, 
No. 8, 60-69.

[20] Qu, L., Liu, Y., Baek, J.B., et al. (2010) Nitrogen-Doped Graphene as Efficient Metal-Free Electrocatalyst for Oxygen Reduction in Fuel Cells. ACS Nano, 4, 1321-1326. https://doi.org/10.1021/nn901850u

[21] Wei, D., Liu, Y., Wang, Y., et al. (2009) Synthesis of N-Doped Graphene by Chemical Vapor Deposition and Its Electrical Properties. Nano Letters, 9, 1752. https://doi.org/10.1021/nl803279t

[22] Su, P., Guo, H., et al. (2012) Preparation of Nitrogen-Doped Graphene and Its Supercapacitive Properties. Chinese Journal of Physical Chemistry, 28, 2745-2753.

[23] Guo, B., Liu, Q., Chen, E., et al. (2010) Controllable N-Doping of Graphene. Nano Letters, 10, 4975. https://doi.org/10.1021/nl103079j

[24] Panchakarla, L.S., Subrahmanyam, K.S., Saha, S.K., et al. (2009) Synthesis, Structure and Properties of Boron and Nitrogen Doped Graphene.

[25] Zhu, L., Zhang, S., Cui, Y., et al. (2013) One Step Synthesis and Capacitive Performance of Graphene Nanosheets/ $\mathrm{Mn}_{3} \mathrm{O}_{4}$ Composite. Electrochimica Acta, 89, 18-23. https://doi.org/10.1016/j.electacta.2012.10.157

[26] Zhao, X., Zhang, L., Murali, S., et al. (2012) Incorporation of Manganese Dioxide within Ultraporous Activated Graphene for High-Performance Electrochemical Capacitors. Acs Nano, 6, 5404-5412. https://doi.org/10.1021/nn3012916

[27] Li, Z., Wang, J., Liu, S., et al. (2011) Synthesis of Hydrothermally Reduced Graphene $/ \mathrm{MnO}_{2}$ Composites and Their Electrochemical Properties as Supercapacitors. Journal of Power Sources, 196, 8160-8165. https://doi.org/10.1016/j.jpowsour.2011.05.036

[28] Rakhi, R.B., Chen, W., Cha, D., et al. (2011) High Performance Supercapacitors Using Metal Oxide Anchored Graphene Nanosheet Electrodes. Journal of Materials Chemistry, 21, 16197-16204. https://doi.org/10.1039/c1jm12963e

[29] Wu, C.H., Deng, S.X., Wang, H., et al. (2014) Preparation of Novel Three Dimensional NiO/Ultrathin Derived Graphene Hybrid for Supercapacitor Applications. Acs Applied Materials \& Interfaces, 6, 1106. https://doi.org/10.1021/am404691w

[30] Wu, Q., Xu, Y., Yao, Z., et al. (2010) Supercapacitors Based on Flexible Graphene/Polyaniline Nanofiber Composite Films. Acs Nano, 4, 1963-1970. https://doi.org/10.1021/nn1000035

[31] Zhang, K., Zhang, L.L., Zhao, X.S., et al. (2010) Graphene/Polyaniline Nanofiber Composites as Supercapacitor Electrodes. Chemistry of Materials, 22, 1392-1401. https://doi.org/10.1021/cm902876u

[32] Yamashiro, S. and Nakamura, K. (2011) Equivalent Circuit Model for Electric Double Layer Capacitors and Its Applications. IEEJ Transactions on Power and Energy, 131, 524-529. https://doi.org/10.1541/ieejpes.131.524

[33] Du, W., Ju, X., et al. (2017) Preparation of Graphene/Polypyrrole Conductive Composite Supercapacitor Electrode. Functional Materials, 48, 1034-1037.

[34] Wang, D.W., Li, F., Zhao, J., et al. (2009) Fabrication of Graphene/Polyaniline Composite Paper via in Situ Anodic Electropolymerization for High-Performance Flexible Electrode. ACS Nano, 3, 1745-1752. https://doi.org/10.1021/nn900297m 